\title{
Asymptotic Behavior of Higher-Order Quasilinear Neutral Differential Equations
}

\author{
Tongxing $\mathrm{Li}^{1}$ and Yuriy V. Rogovchenko ${ }^{2}$ \\ ${ }^{1}$ Qingdao Technological University, Feixian, Shandong 273400, China \\ ${ }^{2}$ Department of Mathematical Sciences, University of Agder, P.O. Box 422, 4604 Kristiansand, Norway \\ Correspondence should be addressed to Yuriy V. Rogovchenko; yuriy.rogovchenko@uia.no
}

Received 29 September 2013; Accepted 17 November 2013; Published 19 January 2014

Academic Editor: Josef Diblik

Copyright (c) $2014 \mathrm{~T}$. Li and Yu. V. Rogovchenko. This is an open access article distributed under the Creative Commons Attribution License, which permits unrestricted use, distribution, and reproduction in any medium, provided the original work is properly cited.

\begin{abstract}
We study asymptotic behavior of solutions to a class of higher-order quasilinear neutral differential equations under the assumptions that allow applications to even- and odd-order differential equations with delayed and advanced arguments, as well as to functional differential equations with more complex arguments that may, for instance, alternate indefinitely between delayed and advanced types. New theorems extend a number of results reported in the literature. Illustrative examples are presented.
\end{abstract}

\section{Introduction}

In this paper, we study asymptotic behavior of solutions to a class of higher-order quasilinear neutral functional differential equations

$$
\left(r(t)\left(z^{(n-1)}(t)\right)^{\alpha}\right)^{\prime}+q(t) x^{\beta}(\sigma(t))=0,
$$

where $t \in \mathbb{\|}:=\left[t_{0}, \infty\right), t_{0} \in \mathbb{R}, z(t):=x(t)+p(t) x(\tau(t))$, $r, \tau, \sigma \in C^{1}(\mathbb{\square}, \mathbb{R}), r^{\prime}(t) \geq 0, r(t)>0, \lim _{t \rightarrow \infty} \sigma(t)=\infty$, $p, q \in \mathrm{C}(\mathbb{\square}, \mathbb{R}), q(t) \geq 0$, and $q(t)$ does not vanish eventually. We also assume that $\alpha, \beta \in \mathfrak{R}$, where $\mathfrak{R}$ stands for the set containing all quotients of odd positive integers. Analysis of qualitative properties of (1) is important not only for the sake of further development of the oscillation theory, but for practical reasons too. In fact, a particular case of (1), an Emden-Fowler type equation

$$
\left(r(t)\left(x^{(n-1)}(t)\right)^{\alpha}\right)^{\prime}+q(t) x^{\beta}(\sigma(t))=0
$$

has numerous applications in physics and engineering; see, for instance, the papers by Ou and Wong [1] or Wong [2].

As customary, by a solution of (1) we understand a function $x \in \mathrm{C}\left(\left[T_{x}, \infty\right), \mathbb{R}\right), T_{x} \geq t_{0}$, which has the property $r\left(z^{(n-1)}\right)^{\alpha} \in \mathrm{C}^{1}\left(\left[T_{x}, \infty\right), \mathbb{R}\right)$ and turns (1) into identity for all $t \in\left[T_{x}, \infty\right)$. We deal only with proper solutions $x$ of (1) that satisfy the condition $\sup \{|x(t)|: t \geq T\}>0$ for all $T \geq T_{x}$ and tacitly assume that (1) possesses such solutions. A solution of (1) is said to be oscillatory if it has arbitrarily large zeros on the ray $\left[T_{x}, \infty\right)$; otherwise, it is termed nonoscillatory. Equation (1) is called oscillatory if all its proper solutions are oscillatory.

For several decades, an increasing interest in obtaining sufficient conditions for oscillatory and nonoscillatory behavior of different classes of differential equations has been observed; see, for instance, the monographs [3-6], the papers $[1,2,7-25]$, and the references cited therein. Let us briefly comment on a number of related results which motivated our study. Questions regarding the oscillation and asymptotic behavior of solutions to (2) have been studied by Džurina and Baculíková [12] and Zhang et al. [23, 25]. In particular, Zhang et al. [23, 25] derived some results on the oscillation and asymptotic behavior of solutions to (2) in the case where $\alpha \geq \beta, \sigma(t)<t$, and

$$
\int_{t_{0}}^{\infty} r^{-1 / \alpha}(t) \mathrm{d} t<\infty .
$$

Oscillation criteria for (1) for $n=2, \alpha=\beta=1$, and $0 \leq$ $p(t) \leq p_{0}<\infty$ can be found in the papers by Baculíková and Džurina [8] and Li et al. [18]. A number of oscillation results for (1) have been established by Baculíková et al. [11] 
and Xing et al. [22] under the assumptions that $\alpha=\beta, 0 \leq$ $p(t) \leq p_{0}<\infty$, and

$$
\int_{t_{0}}^{\infty} r^{-1 / \alpha}(t) \mathrm{d} t=\infty .
$$

We conclude by mentioning that Baculíková and Džurina [10] studied another particular case of (1) assuming that $\alpha=\beta=$ $1,0 \leq p(t) \leq p_{0}<\infty$, and

$$
\int_{t_{0}}^{\infty} r^{-1}(t) \mathrm{d} t=\infty
$$

It should be noted that research in this paper was strongly motivated by the recent contributions of Baculíková and Džurina [10], Li et al. [18], and Zhang et al. [23, 25]. Our principal goal is to analyze the asymptotic behavior of solutions to (1) in the case where condition (3) holds. We provide sufficient conditions which ensure that solutions to (1) are either oscillatory or approach zero at infinity. In some cases, we reveal oscillatory nature of (1). However, we do not discuss in this paper nonoscillation results referring to the recent monograph by Agarwal et al. [3] for an excellent analysis of recent advances in this direction.

As usual, all functional inequalities are supposed to hold for all $t$ large enough. Without loss of generality, we deal only with positive solutions of (1) since, under our assumptions, if $x(t)$ is a solution, then $-x(t)$ is a solution of this equation too.

In the sequel, we denote by $\tau^{-1}$ the function which is inverse to $\tau$. We also adopt the following notation for a compact presentation of our results:

$$
\begin{aligned}
& A(t):=\int_{t}^{\infty} r^{-1 / \alpha}(s) \mathrm{d} s, \\
& Q(t):=\min \{q(t), q(\tau(t))\}, \\
& R(t):=\max \{r(t), r(\tau(t))\}, \\
& Q_{\gamma}(t):=Q(t)\left(\frac{\left(\eta_{1}(t)\right)^{n-1}}{r^{1 / \alpha}\left(\eta_{1}(t)\right)}\right)^{\gamma} \\
& Q_{\beta}(t):=Q(t)\left(\sigma^{n-2}(t)\right)^{\beta} \text {, } \\
& \widetilde{Q}_{\beta}(t):=Q(t)\left(\frac{\left(\eta_{1}(t)\right)^{n-1}}{r^{1 / \beta}\left(\eta_{1}(t)\right)}\right)^{\beta}, \\
& Q_{\theta}(t):=Q(t)\left(\int_{\eta_{3}(t)}^{\infty}\left(\eta-\eta_{3}(t)\right)^{n-3} A(\eta) \mathrm{d} \eta\right)^{\theta}, \\
& \bar{Q}_{\beta}(t):=Q(t)\left(\int_{\eta_{3}(t)}^{\infty}\left(\eta-\eta_{3}(t)\right)^{n-3} A(\eta) \mathrm{d} \eta\right)^{\beta} \text {, } \\
& \widehat{Q}_{\beta}(t):=Q(t)\left(\frac{\sigma^{n-1}(t)}{r^{1 / \beta}(\sigma(t))}\right)^{\beta}, \\
& \widetilde{Q}_{\gamma}(t):=Q(t)\left(\frac{\sigma^{n-1}(t)}{r^{1 / \alpha}(\sigma(t))}\right)^{\gamma},
\end{aligned}
$$

where the meaning of $\gamma, \theta, \eta_{1}$, and $\eta_{3}$ will be explained later.

\section{Asymptotic Behavior of Solutions to Even-Order Equations}

In what follows, $\tau(t)$ can be both a delayed or an advanced argument. Throughout this section, in addition to the basic assumptions listed in the introduction, it is also supposed that (3) holds along with

$$
\begin{aligned}
& \left(H_{1}\right) 0 \leq p(t) \leq p_{0}<\infty, \text { for some constant } p_{0} \\
& \left(H_{2}\right) \tau^{\prime}(t) \geq \tau_{*}>0, \tau \circ \sigma=\sigma \circ \tau .
\end{aligned}
$$

We need the following auxiliary results.

Lemma 1 (see [20]). Let $f \in C^{n}\left(\left[t_{0}, \infty\right), \mathbb{R}^{+}\right)$. If the $n$th derivative $f^{(n)}(t)$ is eventually of one sign for all large $t$, then there exist a $t_{1} \geq t_{0}$ and an integer $l, 0 \leq l \leq n$ with $n+l$ even for $f^{(n)}(t) \geq 0$, or $n+$ lodd for $f^{(n)}(t) \leq 0$ such that

$$
\begin{gathered}
l>0 \text { yields } f^{(k)}(t)>0 \quad \text { for } t \geq t_{1}, k=0,1, \ldots, l-1, \\
l \leq n-1 \text { yields }(-1)^{l+k} f^{(k)}(t)>0 \quad \text { for } t \geq t_{1}, \\
k=l, l+1, \ldots, n-1 .
\end{gathered}
$$

Lemma 2 (see [5, Lemma 2.2.3]). Let $f$ be as in Lemma 1,

$$
f^{(n)}(t) f^{(n-1)}(t) \leq 0
$$

for $t \geq t_{1}$, and assume also that

$$
\lim _{t \rightarrow \infty} f(t) \neq 0 \text {. }
$$

Then, for every constant $\lambda \in(0,1)$, there exists a $t_{\lambda} \in\left[t_{1}, \infty\right)$ such that

$$
f(t) \geq \frac{\lambda}{(n-1) !} t^{n-1}\left|f^{(n-1)}(t)\right|
$$

for all $t \in\left[t_{\lambda}, \infty\right)$.

We are in a position now to state and prove principal results of this paper for even-order equations.

Theorem 3. Let $n \geq 2$ be even and let $0<\beta \leq 1$. Assume that conditions $\left(H_{1}\right)$ and $\left(H_{2}\right)$ are satisfied, and there exist two numbers $\gamma, \lambda \in \Re$ such that $\gamma \leq \beta \leq \lambda$ and $\gamma<\alpha<\lambda$. Suppose further that there exist two functions $\eta_{1}, \eta_{2} \in C(\mathbb{\square}, \mathbb{R})$ such that

$$
\begin{gathered}
\eta_{1}(t) \leq \sigma(t) \leq \eta_{2}(t), \quad \eta_{1}(t)<t \leq \tau(t)<\eta_{2}(t), \\
\lim _{t \rightarrow \infty} \eta_{1}(t)=\infty .
\end{gathered}
$$

If

$$
\begin{gathered}
\int^{\infty} Q_{\gamma}(t) \mathrm{d} t=\infty, \\
\int^{\infty} Q_{\beta}(t) A^{\lambda}\left(\eta_{2}(t)\right) \mathrm{d} t=\infty,
\end{gathered}
$$

every solution $x(t)$ of $(1)$ is either oscillatory or satisfies

$$
\lim _{t \rightarrow \infty} x(t)=0 .
$$


Proof. Assume that (1) has a nonoscillatory solution $x(t)$ which is eventually positive and such that

$$
\lim _{t \rightarrow \infty} x(t) \neq 0 .
$$

Then $z$ satisfies

$$
\begin{aligned}
z(\sigma(t)) & =x(\sigma(t))+p(\sigma(t)) x(\tau(\sigma(t))) \\
& \leq x(\sigma(t))+p_{0} x(\tau(\sigma(t))) .
\end{aligned}
$$

In view of (1), we have

$$
\begin{aligned}
0= & \frac{p_{0}^{\beta}}{\tau^{\prime}(t)}\left(r(\tau(t))\left(z^{(n-1)}(\tau(t))\right)^{\alpha}\right)^{\prime} \\
& +p_{0}^{\beta} q(\tau(t)) x^{\beta}(\sigma(\tau(t))) \\
\geq & \frac{p_{0}^{\beta}}{\tau_{*}}\left(r(\tau(t))\left(z^{(n-1)}(\tau(t))\right)^{\alpha}\right)^{\prime} \\
& +p_{0}^{\beta} q(\tau(t)) x^{\beta}(\sigma(\tau(t))) .
\end{aligned}
$$

Using (16) and [9, Lemma 2], we obtain

$$
\begin{aligned}
q(t) & x^{\beta}(\sigma(t))+p_{0}{ }^{\beta} q(\tau(t)) x^{\beta}(\sigma(\tau(t))) \\
= & q(t) x^{\beta}(\sigma(t))+p_{0}{ }^{\beta} q(\tau(t)) x^{\beta}(\tau(\sigma(t))) \\
& \geq Q(t) z^{\beta}(\sigma(t)) .
\end{aligned}
$$

It follows from (1), (17), and (18) that

$$
\begin{gathered}
\left(r(t)\left(z^{(n-1)}(t)\right)^{\alpha}+\frac{p_{0}^{\beta}}{\tau_{*}} r(\tau(t))\left(z^{(n-1)}(\tau(t))\right)^{\alpha}\right)^{\prime} \\
+Q(t) z^{\beta}(\sigma(t)) \leq 0 .
\end{gathered}
$$

As in the proof of [25, Theorem 2.1], we conclude that, by virtue of (1) and Lemma 1, there are two possibilities, either

$$
\begin{gathered}
z(t)>0, \quad z^{\prime}(t)>0, \quad z^{(n-1)}(t)>0, \\
z^{(n)}(t) \leq 0, \quad\left(r(t)\left(z^{(n-1)}(t)\right)^{\alpha}\right)^{\prime} \leq 0,
\end{gathered}
$$

or

$$
\begin{gathered}
z(t)>0, \quad z^{(n-2)}(t)>0, \\
z^{(n-1)}(t)<0, \quad\left(r(t)\left(z^{(n-1)}(t)\right)^{\alpha}\right)^{\prime} \leq 0,
\end{gathered}
$$

for all $t \geq t_{1}$, where $t_{1} \geq t_{0}$ is large enough.

Case I. Suppose first that conditions (20) hold. Using inequality (19) and assumption $\eta_{1}(t) \leq \sigma(t)$, we conclude that

$$
\begin{aligned}
& \left(r(t)\left(z^{(n-1)}(t)\right)^{\alpha}+\frac{p_{0}^{\beta}}{\tau_{*}} r(\tau(t))\left(z^{(n-1)}(\tau(t))\right)^{\alpha}\right)^{\prime} \\
& +Q(t) z^{\beta}\left(\eta_{1}(t)\right) \leq 0 .
\end{aligned}
$$

Furthermore, by the monotonicity of $z(t)$, there exists a constant $M>0$ such that

$$
z^{\beta}\left(\eta_{1}(t)\right)=z^{\beta-\gamma}\left(\eta_{1}(t)\right) z^{\gamma}\left(\eta_{1}(t)\right) \geq M^{\beta-\gamma} z^{\gamma}\left(\eta_{1}(t)\right) .
$$

Combining (22) and (23), we have

$$
\begin{aligned}
& \left(r(t)\left(z^{(n-1)}(t)\right)^{\alpha}+\frac{p_{0}{ }^{\beta}}{\tau_{*}} r(\tau(t))\left(z^{(n-1)}(\tau(t))\right)^{\alpha}\right)^{\prime} \\
& +M_{1} Q(t) z^{\gamma}\left(\eta_{1}(t)\right) \leq 0,
\end{aligned}
$$

where $M_{1}=M^{\beta-\gamma}$. An application of conditions (20) allows us to deduce that the function

$$
w(t):=r(t)\left(z^{(n-1)}(t)\right)^{\alpha}
$$

is positive and nonincreasing. By Lemma 2, we have

$$
\begin{aligned}
z(t) & \geq \frac{\lambda t^{n-1}}{(n-1) ! r^{1 / \alpha}(t)} r^{1 / \alpha}(t) z^{(n-1)}(t) \\
& =\frac{\lambda t^{n-1}}{(n-1) ! r^{1 / \alpha}(t)} w^{1 / \alpha}(t)
\end{aligned}
$$

for every $\lambda \in(0,1)$ and for all sufficiently large $t$. Using (26) in (24), we conclude that $w(t)$ is a positive solution of a delay differential inequality

$$
\begin{aligned}
(w(t) & \left.+\frac{p_{0}{ }^{\beta}}{\tau_{*}} w(\tau(t))\right)^{\prime} \\
& +M_{1}\left(\frac{\lambda}{(n-1) !}\right)^{\gamma} Q_{\gamma}(t) w^{\gamma / \alpha}\left(\eta_{1}(t)\right) \leq 0 .
\end{aligned}
$$

Define now a function $y(t)$ by

$$
y(t):=w(t)+\frac{p_{0}^{\beta}}{\tau_{*}} w(\tau(t))
$$

Then, by the monotonicity of $w(t)$,

$$
y(t) \leq w(t)\left(1+\frac{p_{0}^{\beta}}{\tau_{*}}\right) .
$$

Substituting (29) into (27), we observe that $y(t)$ is a positive solution of a delay differential inequality

$$
y^{\prime}(t)+M_{1}\left(\frac{\lambda}{(n-1) !}\right)^{\gamma}\left(\frac{\tau_{*}}{\tau_{*}+p_{0}^{\beta}}\right)^{\gamma / \alpha} Q_{\gamma}(t) y^{\gamma / \alpha}\left(\eta_{1}(t)\right) \leq 0 .
$$

Then, by virtue of [21, Theorem 1], the associated delay differential equation

$$
y^{\prime}(t)+M_{1}\left(\frac{\lambda}{(n-1) !}\right)^{\gamma}\left(\frac{\tau_{*}}{\tau_{*}+p_{0} \beta}\right)^{\gamma / \alpha} Q_{\gamma}(t) y^{\gamma / \alpha}\left(\eta_{1}(t)\right)=0
$$


also has a positive solution. However, the result by Kitamura and Kusano [15, Theorem 2] implies that, under assumption (12), (31) is oscillatory. Therefore, (1) cannot have positive solutions.

Case II. Assume now that conditions (21) hold. By virtue of (15), we have that

$$
\lim _{t \rightarrow \infty} z(t) \neq 0 .
$$

An application of Lemma 2 yields

$$
z(t) \geq \frac{\lambda}{(n-2) !} t^{n-2} z^{(n-2)}(t),
$$

for any $\lambda \in(0,1)$ and for all sufficiently large $t$. Hence, by (19) and (33), we obtain

$$
\begin{gathered}
\left(r(t)\left(z^{(n-1)}(t)\right)^{\alpha}+\frac{p_{0}^{\beta}}{\tau_{*}} r(\tau(t))\left(z^{(n-1)}(\tau(t))\right)^{\alpha}\right)^{\prime} \\
+\left(\frac{\lambda}{(n-2) !}\right)^{\beta} Q_{\beta}(t)\left(z^{(n-2)}(\sigma(t))\right)^{\beta} \leq 0
\end{gathered}
$$

Using conditions $z^{(n-1)}(t)<0, \sigma(t) \leq \eta_{2}(t)$, and inequality (34), we have

$$
\begin{gathered}
\left(r(t)\left(z^{(n-1)}(t)\right)^{\alpha}+\frac{p_{0}^{\beta}}{\tau_{*}} r(\tau(t))\left(z^{(n-1)}(\tau(t))\right)^{\alpha}\right)^{\prime} \\
+\left(\frac{\lambda}{(n-2) !}\right)^{\beta} Q_{\beta}(t)\left(z^{(n-2)}\left(\eta_{2}(t)\right)\right)^{\beta} \leq 0
\end{gathered}
$$

Furthermore, by the monotonicity of $z^{(n-2)}(t)$, there exists a constant $N>0$ such that

$$
\begin{aligned}
\left(z^{(n-2)}\left(\eta_{1}(t)\right)\right)^{\beta} & =\left(z^{(n-2)}\left(\eta_{1}(t)\right)\right)^{\beta-\lambda}\left(z^{(n-2)}\left(\eta_{1}(t)\right)\right)^{\lambda} \\
& \geq N^{\beta-\lambda}\left(z^{(n-2)}\left(\eta_{2}(t)\right)\right)^{\lambda} .
\end{aligned}
$$

Combining (35) and (36), we arrive at

$$
\begin{array}{r}
\left(r(t)\left(z^{(n-1)}(t)\right)^{\alpha}+\frac{p_{0}{ }^{\beta}}{\tau_{*}} r(\tau(t))\left(z^{(n-1)}(\tau(t))\right)^{\alpha}\right)^{\prime} \\
+N_{1}\left(\frac{\lambda}{(n-2) !}\right)^{\beta} Q_{\beta}(t)\left(z^{(n-2)}\left(\eta_{2}(t)\right)\right)^{\lambda} \leq 0,
\end{array}
$$

where $N_{1}=N^{\beta-\lambda}$. Using the monotonicity of $w(t)$, for $s \geq$ $t \geq t_{1}$, we conclude that

$$
r^{1 / \alpha}(s) z^{(n-1)}(s) \leq r^{1 / \alpha}(t) z^{(n-1)}(t) .
$$

Dividing (38) by $r^{1 / \alpha}(s)$ and integrating the resulting inequality from $t$ to $l$, we obtain

$$
\begin{aligned}
& z^{(n-2)}(l) \\
& \quad \leq z^{(n-2)}(t)+r^{1 / \alpha}(t) z^{(n-1)}(t) \int_{t}^{l} r^{-1 / \alpha}(s) \mathrm{d} s .
\end{aligned}
$$

Passing to the limit as $l \rightarrow \infty$, we deduce that

$$
0 \leq z^{(n-2)}(t)+r^{1 / \alpha}(t) z^{(n-1)}(t) A(t)
$$

which yields

$$
z^{(n-2)}(t) \geq-A(t) r^{1 / \alpha}(t) z^{(n-1)}(t)=-A(t) w^{1 / \alpha}(t) .
$$

Combining (37) and (41), we have

$$
\begin{aligned}
& \left(r(t)\left(z^{(n-1)}(t)\right)^{\alpha}+\frac{p_{0}{ }^{\beta}}{\tau_{*}} r(\tau(t))\left(z^{(n-1)}(\tau(t))\right)^{\alpha}\right)^{\prime} \\
& \quad-N_{1}\left(\frac{\lambda}{(n-2) !}\right)^{\beta} Q_{\beta}(t) A^{\lambda}\left(\eta_{2}(t)\right) w^{\lambda / \alpha}\left(\eta_{2}(t)\right) \leq 0 .
\end{aligned}
$$

Using again monotonicity of $w(t)$, we conclude that

$$
y(t) \geq w(\tau(t))\left(1+\frac{p_{0}{ }^{\beta}}{\tau_{*}}\right) .
$$

Substituting (43) into (42), we observe that $y(t)$ is a negative solution of an advanced differential inequality

$$
\begin{aligned}
y^{\prime}(t) & -N_{1}\left(\frac{\lambda}{(n-2) !}\right)^{\beta}\left(\frac{\tau_{*}}{\tau_{*}+p_{0}^{\beta}}\right)^{\lambda / \alpha} \\
& \times Q_{\beta}(t) A^{\lambda}\left(\eta_{2}(t)\right) y^{\lambda / \alpha}\left(\tau^{-1}\left(\eta_{2}(t)\right)\right) \leq 0,
\end{aligned}
$$

which implies that $u(t):=-y(t)$ is a positive solution of an advanced differential inequality

$$
\begin{aligned}
u^{\prime}(t) & -N_{1}\left(\frac{\lambda}{(n-2) !}\right)^{\beta}\left(\frac{\tau_{*}}{\tau_{*}+p_{0} \beta}\right)^{\lambda / \alpha} \\
& \times Q_{\beta}(t) A^{\lambda}\left(\eta_{2}(t)\right) u^{\lambda / \alpha}\left(\tau^{-1}\left(\eta_{2}(t)\right)\right) \geq 0 .
\end{aligned}
$$

Consequently, by [7, Lemma 2.3], the associated advanced differential equation

$$
\begin{aligned}
u^{\prime}(t) & -N_{1}\left(\frac{\lambda}{(n-2) !}\right)^{\beta}\left(\frac{\tau_{*}}{\tau_{*}+p_{0}^{\beta}}\right)^{\lambda / \alpha} \\
& \times Q_{\beta}(t) A^{\lambda}\left(\eta_{2}(t)\right) u^{\lambda / \alpha}\left(\tau^{-1}\left(\eta_{2}(t)\right)\right)=0
\end{aligned}
$$

also has a positive solution. However, it follows from [15, Theorem 1] that if condition (13) holds, (46) is oscillatory. Therefore, (1) cannot have positive solutions. This contradiction with our initial assumption completes the proof.

Theorem 4. Let $n \geq 2$ be even, and let $0<\alpha=\beta \leq 1$. Assume that conditions $\left(H_{1}\right)$ and $\left(H_{2}\right)$ hold, and there exist 
two functions $\eta_{1}, \eta_{2} \in C(\mathbb{Q}, \mathbb{R})$ satisfying (11). Suppose also that conditions

$$
\begin{aligned}
& \frac{\tau_{*}}{((n-1) !)^{\beta}\left(\tau_{*}+p_{0} \beta\right)} \liminf _{t \rightarrow \infty} \int_{\eta_{1}(t)}^{t} \widetilde{Q}_{\beta}(s) \mathrm{d} s>\frac{1}{e}, \\
& \frac{\tau_{*}}{((n-2) !)^{\beta}\left(\tau_{*}+p_{0} \beta\right)} \liminf _{t \rightarrow \infty} \int_{t}^{\tau^{-1}\left(\eta_{2}(t)\right)} Q_{\beta}(s) A^{\beta}\left(\eta_{2}(s)\right) \mathrm{d} s \\
& >\frac{1}{e}
\end{aligned}
$$

are satisfied. Then conclusion of Theorem 3 remains intact.

Proof. Assume that $x(t)$ is an eventually positive solution of (1) that satisfies (15). Proceeding as in the proof of Theorem 3, one comes to the conclusion that, for every $\lambda \in(0,1)$, a delay differential equation

$$
y^{\prime}(t)+\left(\frac{\lambda}{(n-1) !}\right)^{\beta} \frac{\tau_{*}}{\tau_{*}+p_{0} \beta} \widetilde{Q}_{\beta}(t) y\left(\eta_{1}(t)\right)=0
$$

and an advanced differential equation

$$
\begin{aligned}
u^{\prime}(t) & -\left(\frac{\lambda}{(n-2) !}\right)^{\beta} \frac{\tau_{*}}{\tau_{*}+p_{0} \beta} Q_{\beta}(t) A^{\beta}\left(\eta_{2}(t)\right) \\
& \times u\left(\tau^{-1}\left(\eta_{2}(t)\right)\right)=0
\end{aligned}
$$

both have positive solutions. On the other hand, condition (47) and [9, Lemma 4] imply that (49) is oscillatory, a contradiction. Likewise, by virtue of [6, Theorem 2.4.1], condition (48) yields that (50) has no positive solutions. This contradiction completes the proof.

Theorem 5. Let $n \geq 2$ be even and $0<\beta \leq 1$. Assume that conditions $\left(H_{1}\right)$ and $\left(H_{2}\right)$ are satisfied, and there exist two numbers $\gamma, \lambda \in \mathfrak{R}$ as in Theorem 3 and two functions $\eta_{1}, \eta_{2} \in C(\mathbb{\square}, \mathbb{R})$ such that

$$
\begin{gathered}
\eta_{1}(t) \leq \sigma(t) \leq \eta_{2}(t), \quad \eta_{1}(t)<\tau(t) \leq t<\eta_{2}(t), \\
\lim _{t \rightarrow \infty} \eta_{1}(t)=\infty
\end{gathered}
$$

If conditions (12) and (13) hold, the conclusion of Theorem 3 remains intact.

Proof. As above, let $x(t)$ be an eventually positive solution of (1) that satisfies (15). As in the proof of Theorem 3, we split the argument into two parts.

Case I. Assume first that (20) is satisfied. It has been established in the proof of Theorem 3 that the function $w(t)$ defined by (25) is positive, nonincreasing, and satisfies inequality (27). Introducing again $y(t)$ by (28) and using the monotonicity of $w(t)$, we conclude that

$$
y(t) \leq w(\tau(t))\left(1+\frac{p_{0}^{\beta}}{\tau_{*}}\right) .
$$

Substitution of (52) into (27) implies that, for sufficiently large $t, y(t)$ is a positive solution of a delay differential inequality

$$
\begin{aligned}
y^{\prime}(t) & +M_{1}\left(\frac{\lambda}{(n-1) !}\right)^{\gamma}\left(\frac{\tau_{*}}{\tau_{*}+p_{0} \beta}\right)^{\gamma / \alpha} Q_{\gamma}(t) \\
& \times y^{\gamma / \alpha}\left(\tau^{-1}\left(\eta_{1}(t)\right)\right) \leq 0 .
\end{aligned}
$$

Then, by virtue of [21, Theorem 1], the associated delay differential equation

$$
\begin{aligned}
y^{\prime}(t) & +M_{1}\left(\frac{\lambda}{(n-1) !}\right)^{\gamma}\left(\frac{\tau_{*}}{\tau_{*}+p_{0}^{\beta}}\right)^{\gamma / \alpha} Q_{\gamma}(t) \\
& \times y^{\gamma / \alpha}\left(\tau^{-1}\left(\eta_{1}(t)\right)\right)=0
\end{aligned}
$$

also has a positive solution. However, [15, Theorem 2] implies that if (12) holds, (54) is oscillatory. Therefore, (1) cannot have positive solutions.

Case II. Assume now that (21) is satisfied. It has been established in the proof of Theorem 3 that the function $w(t)$ defined by (25) is negative, nonincreasing, and satisfies the inequality (42). Introducing again $y(t)$ by (28) and using the monotonicity of $w(t)$, we conclude that

$$
y(t) \geq w(t)\left(1+\frac{p_{0}^{\beta}}{\tau_{*}}\right) .
$$

Substituting (55) into (42), we observe that $y(t)$ is a negative solution of an advanced differential inequality

$$
\begin{aligned}
y^{\prime}(t) & -N_{1}\left(\frac{\lambda}{(n-2) !}\right)^{\beta}\left(\frac{\tau_{*}}{\tau_{*}+p_{0} \beta}\right)^{\lambda / \alpha} Q_{\beta}(t) \\
& \times A^{\lambda}\left(\eta_{2}(t)\right) y^{\lambda / \alpha}\left(\eta_{2}(t)\right) \leq 0 .
\end{aligned}
$$

That is, $u(t):=-y(t)$ is a positive solution of an advanced differential inequality

$$
\begin{aligned}
u^{\prime}(t) & -N_{1}\left(\frac{\lambda}{(n-2) !}\right)^{\beta}\left(\frac{\tau_{*}}{\tau_{*}+p_{0} \beta}\right)^{\lambda / \alpha} Q_{\beta}(t) \\
& \times A^{\lambda}\left(\eta_{2}(t)\right) u^{\lambda / \alpha}\left(\eta_{2}(t)\right) \geq 0
\end{aligned}
$$

Then, by [7, Lemma 2.3], the associated advanced differential equation

$$
\begin{aligned}
u^{\prime}(t) & -N_{1}\left(\frac{\lambda}{(n-2) !}\right)^{\beta}\left(\frac{\tau_{*}}{\tau_{*}+p_{0}^{\beta}}\right)^{\lambda / \alpha} Q_{\beta}(t) \\
& \times A^{\lambda}\left(\eta_{2}(t)\right) u^{\lambda / \alpha}\left(\eta_{2}(t)\right)=0
\end{aligned}
$$

also has a positive solution. However, [15, Theorem 1] implies that, under assumption (13), (58) is oscillatory. Therefore, (1) cannot have positive solutions. This contradiction with our initial assumption completes the proof. 
Theorem 6. Let $n \geq 2$ be even and $0<\alpha=\beta \leq 1$. Assume that conditions $\left(H_{1}\right)$ and $\left(H_{2}\right)$ are satisfied, and there exist two functions $\eta_{1}, \eta_{2} \in C(\mathbb{Q}, \mathbb{R})$ satisfying (51). Suppose also that

$$
\begin{aligned}
& \frac{\tau_{*}}{((n-1) !)^{\beta}\left(\tau_{*}+p_{0}^{\beta}\right)} \liminf _{t \rightarrow \infty} \int_{\tau^{-1}\left(\eta_{1}(t)\right)}^{t} \widetilde{Q}_{\beta}(s) \mathrm{d} s>\frac{1}{\mathrm{e}}, \quad(59) \\
& \frac{\tau_{*}}{((n-2) !)^{\beta}\left(\tau_{*}+p_{0}^{\beta}\right)} \liminf _{t \rightarrow \infty} \int_{t}^{\eta_{2}(t)} \mathrm{Q}_{\beta}(s) A^{\beta}\left(\eta_{2}(s)\right) \mathrm{d} s>\frac{1}{\mathrm{e}} .
\end{aligned}
$$

Then the conclusion of Theorem 3 remains intact.

Proof. Assuming that $x(t)$ is an eventually positive solution of (1) that satisfies (15) and proceeding as in the proof of Theorem 5 , one concludes that, for every $\lambda \in(0,1)$, a delay differential equation

$$
y^{\prime}(t)+\left(\frac{\lambda}{(n-1) !}\right)^{\beta} \frac{\tau_{*}}{\tau_{*}+p_{0} \beta} \widetilde{Q}_{\beta}(t) y\left(\tau^{-1}\left(\eta_{1}(t)\right)\right)=0
$$

and an advanced differential equation

$$
u^{\prime}(t)-\frac{\tau_{*}}{\tau_{*}+p_{0} \beta}\left(\frac{\lambda}{(n-2) !}\right)^{\beta} Q_{\beta}(t) A^{\beta}\left(\eta_{2}(t)\right) u\left(\eta_{2}(t)\right)=0
$$

have positive solutions. On the other hand, application of condition (59) along with [9, Lemma 4] implies that (61) is oscillatory, a contradiction. Likewise, by virtue of $[6$, Theorem 2.4.1], condition (60) yields that (62) has no positive solutions. This contradiction completes the proof.

Note that Theorems 3-6 ensure that every solution $x(t)$ of (1) is either oscillatory or tends to zero as $t \rightarrow \infty$ and, unfortunately, cannot distinguish solutions with different behaviors. In the remaining part of this section, we establish several results which guarantee that all solutions of (1) are oscillatory.

Theorem 7. Let $n \geq 4$ be even and $0<\beta \leq 1$. Assume that conditions $\left(H_{1}\right)$ and $\left(H_{2}\right)$ are satisfied, and there exist three numbers $\gamma, \lambda, \theta \in \mathfrak{R}$ such that $\gamma \leq \beta \leq \lambda, \gamma<\alpha<\lambda, \theta \geq$ $\beta$, and $\theta>\alpha$. Suppose further that there exist three functions $\eta_{1}, \eta_{2}, \eta_{3} \in C(\mathbb{Q}, \mathbb{R})$

$$
\eta_{3}(t) \geq \sigma(t), \quad \eta_{3}(t)>\tau(t)
$$

and such that (11) holds. Assume that conditions (12), (13), and

$$
\int^{\infty} Q_{\theta}(t) \mathrm{d} t=\infty
$$

hold. Then (1) is oscillatory.

Proof. Without loss of generality, suppose that $x(t)$ is a nonoscillatory solution of (1) which is eventually positive. As in the proof of Theorem 3, we obtain (19). Applying the same argument as in the paper by Zhang et al. [23, Theorem 2.1], we conclude that, by virtue of (1) and Lemma 1, in addition to the case (20), there are two more possible types of behavior of solutions for $t \geq t_{1}$, where $t_{1} \geq t_{0}$ is large enough in the proof of Theorem 3. Namely, one can also have

$$
\begin{aligned}
& z(t)>0, \quad z^{\prime}(t)>0, \quad z^{(n-2)}(t)>0, \\
& z^{(n-1)}(t)<0, \quad\left(r(t)\left(z^{(n-1)}(t)\right)^{\alpha}\right)^{\prime} \leq 0,
\end{aligned}
$$

or

$$
\begin{aligned}
& z(t)>0, \quad z^{(j)}(t)<0, \quad z^{(j+1)}(t)>0, \\
& z^{(n-1)}(t)<0, \quad\left(r(t)\left(z^{(n-1)}(t)\right)^{\alpha}\right)^{\prime} \leq 0,
\end{aligned}
$$

for all odd integers $j \in\{1,2, \ldots, n-3\}$. However, conditions (12) and (13) yield that neither (20) nor (65) is possible.

Therefore, we have to analyze the only remaining case, and we assume now that all the conditions in (66) are satisfied. Then, inequality (41) holds. Integrating (41) from $t$ to $\infty n-2$ times, we obtain

$$
\begin{aligned}
z(t) & \geq-\frac{\int_{t}^{\infty}(\eta-t)^{n-3} A(\eta) \mathrm{d} \eta}{(n-3) !} r^{1 / \alpha}(t) z^{(n-1)}(t) \\
& =-\frac{\int_{t}^{\infty}(\eta-t)^{n-3} A(\eta) \mathrm{d} \eta}{(n-3) !} w^{1 / \alpha}(t),
\end{aligned}
$$

where $w(t)$ is defined by (25). Taking into account that $z^{\prime}(t)<$ $0, \sigma(t) \leq \eta_{3}(t)$, and using (19), we have

$$
\begin{aligned}
& \left(r(t)\left(z^{(n-1)}(t)\right)^{\alpha}+\frac{p_{0}^{\beta}}{\tau_{*}} r(\tau(t))\left(z^{(n-1)}(\tau(t))\right)^{\alpha}\right)^{\prime} \\
& +Q(t) z^{\beta}\left(\eta_{3}(t)\right) \leq 0 .
\end{aligned}
$$

By virtue of monotonicity of $z(t)$, there exists a constant $M_{2}>$ 0 such that

$$
z^{\beta}\left(\eta_{3}(t)\right)=z^{\beta-\theta}\left(\eta_{3}(t)\right) z^{\theta}\left(\eta_{3}(t)\right) \geq M_{2} z^{\theta}\left(\eta_{3}(t)\right) .
$$

Combining (68) and (69), we obtain

$$
\begin{aligned}
& \left(r(t)\left(z^{(n-1)}(t)\right)^{\alpha}+\frac{p_{0}^{\beta}}{\tau_{*}} r(\tau(t))\left(z^{(n-1)}(\tau(t))\right)^{\alpha}\right)^{\prime} \\
& +M_{2} Q(t) z^{\theta}\left(\eta_{3}(t)\right) \leq 0 .
\end{aligned}
$$

Using (67) in (70), we conclude that in this case, the function $w(t)$ defined by (25) is negative, nonincreasing, and satisfies the inequality

$$
\begin{gathered}
\left(r(t)\left(z^{(n-1)}(t)\right)^{\alpha}+\frac{p_{0}^{\beta}}{\tau_{*}} r(\tau(t))\left(z^{(n-1)}(\tau(t))\right)^{\alpha}\right)^{\prime} \\
-\frac{M_{2}}{((n-3) !)^{\theta}} Q_{\theta}(t) w^{\theta / \alpha}\left(\eta_{3}(t)\right) \leq 0 .
\end{gathered}
$$


Introducing again $y(t)$ by (28) and using the monotonicity of $w(t)$, we arrive at (43). Substitution of (43) into (71) leads to the conclusion that $y(t)$ is a negative solution of an advanced differential inequality

$$
\begin{aligned}
y^{\prime}(t) & -\frac{M_{2}}{((n-3) !)^{\theta}}\left(\frac{\tau_{*}}{\tau_{*}+p_{0}^{\beta}}\right)^{\theta / \alpha} Q_{\theta}(t) y^{\theta / \alpha}\left(\tau^{-1}\left(\eta_{3}(t)\right)\right) \\
& \leq 0
\end{aligned}
$$

in which case the function $u(t):=-y(t)$ is a positive solution of an advanced differential inequality

$$
\begin{aligned}
u^{\prime}(t) & -\frac{M_{2}}{((n-3) !)^{\theta}}\left(\frac{\tau_{*}}{\tau_{*}+p_{0} \beta}\right)^{\theta / \alpha} Q_{\theta}(t) u^{\theta / \alpha}\left(\tau^{-1}\left(\eta_{3}(t)\right)\right) \\
& \geq 0
\end{aligned}
$$

Then, by [7, Lemma 2.3], the associated advanced differential equation

$$
\begin{aligned}
u^{\prime}(t) & -\frac{M_{2}}{((n-3) !)^{\theta}}\left(\frac{\tau_{*}}{\tau_{*}+p_{0}^{\beta}}\right)^{\theta / \alpha} Q_{\theta}(t) u^{\theta / \alpha}\left(\tau^{-1}\left(\eta_{3}(t)\right)\right) \\
& =0
\end{aligned}
$$

also has a positive solution. However, [15, Theorem 1] implies that (74) is oscillatory under assumption (64). Therefore, (1) cannot have positive solutions. This contradiction with our initial assumption completes the proof.

Theorem 8. Let $n \geq 4$ be even and $0<\alpha=\beta \leq 1$. Assume that conditions $\left(H_{1}\right)$ and $\left(H_{2}\right)$ are satisfied, and there exist three functions $\eta_{1}, \eta_{2}, \eta_{3} \in C(\mathbb{\square}, \mathbb{R})$ as in Theorem 7 . Suppose also that conditions (47) and (48) hold. If

$$
\frac{\tau_{*}}{((n-3) !)^{\beta}\left(\tau_{*}+p_{0}^{\beta}\right)} \liminf _{t \rightarrow \infty} \int_{t}^{\tau^{-1}\left(\eta_{3}(t)\right)} \bar{Q}_{\beta}(s) \mathrm{d} s>\frac{1}{\mathrm{e}},
$$

(1) is oscillatory.

Proof. Let $x(t)$ be a nonoscillatory solution of (1) which is eventually positive. As in the proof of Theorem 7, one can have either (20) or (65), or (66). However, conditions (47) and (48) exclude cases (20) and (65). Thus, all the inequalities in (66) should be satisfied. Along the same lines as in the proof of Theorem 7 , one comes to the conclusion that an advanced differential equation

$$
u^{\prime}(t)-\frac{\tau_{*}}{((n-3) !)^{\beta}\left(\tau_{*}+p_{0}^{\beta}\right)} \bar{Q}_{\beta}(t) u\left(\tau^{-1}\left(\eta_{3}(t)\right)\right)=0
$$

has positive solutions. On the other hand, if condition (75) holds, a well-known result [6, Theorem 2.4.1] implies that (76) has no positive solutions. This contradiction completes the proof.
Theorem 9. Let $n \geq 4$ be even, $0<\beta \leq 1$, and assume that conditions $\left(H_{1}\right)$ and $\left(H_{2}\right)$ are satisfied. Suppose further that there exist three numbers $\gamma, \lambda, \theta \in \mathfrak{R}$ as in Theorem 7 and three functions $\eta_{1}, \eta_{2}, \eta_{3} \in C(\mathbb{\square}, \mathbb{R})$ such that (51) is satisfied, $\eta_{3}(t) \geq \sigma(t)$, and $\eta_{3}(t)>t$. If (12), (13), and (64) hold, (1) is oscillatory.

Proof. Let $x(t)$ be an eventually positive nonoscillatory solution of (1). The same argument as in the proof of Theorem 7 yields that (66) holds. Define the function $w(t)$ by (25). From the proof of Theorem 7, we already know that $w(t)$ is negative, nonincreasing, and satisfies the inequality (71). Introducing then the function $y(t)$ by (28) and using the monotonicity of $w(t)$, we arrive at (55). Substituting (55) into (71), we observe that $y(t)$ is a negative solution of an advanced differential inequality

$$
y^{\prime}(t)-\frac{M_{2}}{((n-3) !)^{\theta}}\left(\frac{\tau_{*}}{\tau_{*}+p_{0}^{\beta}}\right)^{\theta / \alpha} Q_{\theta}(t) y^{\theta / \alpha}\left(\eta_{3}(t)\right) \leq 0,
$$

while $u(t):=-y(t)$ is a positive solution of an advanced differential inequality

$$
u^{\prime}(t)-\frac{M_{2}}{((n-3) !)^{\theta}}\left(\frac{\tau_{*}}{\tau_{*}+p_{0}^{\beta}}\right)^{\theta / \alpha} Q_{\theta}(t) u^{\theta / \alpha}\left(\eta_{3}(t)\right) \geq 0
$$

In this case, the result due to Baculíková [7, Lemma 2.3] allows one to deduce that the associated advanced differential equation

$$
u^{\prime}(t)-\frac{M_{2}}{((n-3) !)^{\theta}}\left(\frac{\tau_{*}}{\tau_{*}+p_{0} \beta}\right)^{\theta / \alpha} Q_{\theta}(t) u^{\theta / \alpha}\left(\eta_{3}(t)\right)=0
$$

also has a positive solution. However, it has been established by Kitamura and Kusano [15, Theorem 1] that if condition (64) is satisfied, (79) is oscillatory. Therefore, (1) cannot have positive solutions, and this contradiction with the assumptions of the theorem completes the proof.

Theorem 10. Let $n \geq 4$ be even and $0<\alpha=\beta \leq 1$. Assume that conditions $\left(H_{1}\right)$ and $\left(H_{2}\right)$ are satisfied, and there exist three functions $\eta_{1}, \eta_{2}, \eta_{3} \in C(\mathbb{\square}, \mathbb{R})$ as in Theorem 9. Suppose further that (59), (60) hold, and

$$
\frac{\tau_{*}}{((n-3) !)^{\beta}\left(\tau_{*}+p_{0}^{\beta}\right)} \liminf _{t \rightarrow \infty} \int_{t}^{\eta_{3}(t)} \bar{Q}_{\beta}(s) \mathrm{d} s>\frac{1}{e} .
$$

Then (1) is oscillatory.

Proof. Assuming that $x(t)$ is an eventually positive nonoscillatory solution of (1) and reasoning as in the proof of Theorem 7, one concludes that (66) holds. As in the proof of Theorem 9, we observe that an advanced differential equation

$$
u^{\prime}(t)-\frac{\tau_{*}}{((n-3) !)^{\beta}\left(\tau_{*}+p_{0}^{\beta}\right)} \bar{Q}_{\beta}(t) u\left(\eta_{3}(t)\right)=0
$$


has positive solutions. On the other hand, if condition (80) is satisfied, a result reported by Ladde et al. [6, Theorem 2.4.1] yields that (81) has no positive solutions. This contradiction completes the proof.

\section{Asymptotic Behavior of Solutions to Odd-Order Equations}

In this section, in addition to conditions $\left(H_{1}\right),\left(H_{2}\right)$, and (3), we also assume that

$$
\left(H_{3}\right) \sigma(t)<t
$$

The validity of the following four propositions can be established in the same manner as it has been done for Theorems 3-6. Therefore, to avoid unnecessary repetition, we only formulate counterparts of Theorems 3-6 for the case of odd-order equations.

Theorem 11. Let $n \geq 3$ be odd and let $0<\beta \leq 1$. Assume that conditions $\left(H_{1}\right)-\left(H_{3}\right)$ are satisfied, and there exist two numbers $\gamma, \lambda \in \Re$ as in Theorem 3 and a function $\eta_{4} \in C(\mathbb{Q}, \mathbb{R})$ such that $t \leq \tau(t)<\eta_{4}(t)$. Suppose further that

$$
\begin{gathered}
\int^{\infty} \widetilde{Q}_{\gamma}(t) \mathrm{d} t=\infty, \\
\int^{\infty} Q_{\beta}(t) A^{\lambda}\left(\eta_{4}(t)\right) \mathrm{d} t=\infty .
\end{gathered}
$$

Then the conclusion of Theorem 3 remains intact.

Theorem 12. Let $n \geq 3$ be odd, and let $0<\alpha=\beta \leq 1$. Assume that conditions $\left(H_{1}\right)-\left(H_{3}\right)$ are satisfied, and there exists a function $\eta_{4} \in C(\mathbb{\square}, \mathbb{R})$ as in Theorem 11. Suppose also that

$$
\begin{gathered}
\frac{\tau_{*}}{((n-1) !)^{\beta}\left(\tau_{*}+p_{0}^{\beta}\right)} \liminf _{t \rightarrow \infty} \int_{\sigma(t)}^{t} \widehat{\mathrm{Q}}_{\beta}(s) \mathrm{d} s>\frac{1}{\mathrm{e}}, \\
\frac{\tau_{*}}{((n-2) !)^{\beta}\left(\tau_{*}+p_{0}^{\beta}\right)} \liminf _{t \rightarrow \infty} \int_{t}^{\tau^{-1}\left(\eta_{4}(t)\right)} \mathrm{Q}_{\beta}(s) \\
\times A^{\beta}\left(\eta_{4}(s)\right) d s>\frac{1}{\mathrm{e}} .
\end{gathered}
$$

Then the conclusion of Theorem 3 remains intact.

Theorem 13. Let $n \geq 3$ be odd and let $0<\beta \leq 1$. Assume that conditions $\left(H_{1}\right)-\left(H_{3}\right)$ are satisfied, and there exist two numbers $\gamma, \lambda \in \Re$ as in Theorem 3 and a function $\eta_{4} \in C(\mathbb{\square}, \mathbb{R})$ such that $\sigma(t)<\tau(t) \leq t<\eta_{4}(t)$. Suppose further that conditions (82) are satisfied. Then the conclusion of Theorem 3 remains intact.
Theorem 14. Let $n \geq 3$ be odd, and let $0<\alpha=\beta \leq$ 1. Assume that conditions $\left(H_{1}\right)-\left(H_{3}\right)$ are satisfied, and there exists a function $\eta_{4} \in C(\mathbb{\square}, \mathbb{R})$ as in Theorem 13. If

$$
\begin{gathered}
\frac{\tau_{*}}{((n-1) !)^{\beta}\left(\tau_{*}+p_{0}^{\beta}\right)} \liminf _{t \rightarrow \infty} \int_{\tau^{-1}(\sigma(t))}^{t} \widehat{Q}_{\beta}(s) \mathrm{d} s>\frac{1}{\mathrm{e}}, \\
\frac{\tau_{*}}{((n-2) !)^{\beta}\left(\tau_{*}+p_{0}^{\beta}\right)} \liminf _{t \rightarrow \infty} \int_{t}^{\eta_{4}(t)} Q_{\beta}(s) A^{\beta}\left(\eta_{4}(s)\right) \mathrm{d} s>\frac{1}{\mathrm{e}},
\end{gathered}
$$

the conclusion of Theorem 3 remains intact.

Note that Theorems 11-14 apply only if $\sigma$ is a delayed argument, $\sigma(t)<t$. Hence, it is important to complement such results with the following theorems that can be applied in the case where $\sigma$ is an advanced argument, $\sigma(t) \geq t$.

Theorem 15. Let $n \geq 3$ be odd and let $0<\beta \leq 1$. Assume that conditions $\left(H_{1}\right)$ and $\left(H_{2}\right)$ are satisfied, and there exist two numbers $\gamma, \lambda \in \mathfrak{R}$ as in Theorem 3 and two functions $\eta_{1}, \eta_{2} \in$ $C(\mathbb{\square}, \mathbb{R})$ satisfying (11). Suppose also that

$$
\int_{t_{0}}^{\infty} \xi^{n-2}\left[\frac{1}{R(\xi)} \int_{\xi}^{\infty} \mathrm{Q}(s) \mathrm{d} s\right]^{1 / \alpha} \mathrm{d} \xi=\infty .
$$

If (12) and (13) are satisfied, the conclusion of Theorem 3 remains intact.

Proof. Assume that (1) has an eventually positive solution $x(t)$ satisfying (15). Proceeding as in the proof of Theorem 3, we arrive at (19) and observe that (1) yields that either (20) or (21) holds.

Indeed, it follows from the condition $\left(r(t)\left(z^{(n-1)}(t)\right)^{\alpha}\right)^{\prime} \leq$ 0 that either $z^{(n-1)}(t)>0$ or $z^{(n-1)}(t)<0$. Assume first that $z^{(n-1)}(t)<0$; this immediately leads us to conditions (21). On the other hand, if $z^{(n-1)}(t)>0$, then $z^{(n)}(t) \leq 0$ due to the fact that $r^{\prime}(t) \geq 0$. We claim that $z^{\prime}(t)>0$ eventually. In fact, if this is not the case, then $z^{\prime}(t)<0$ eventually. Since $z(t)>0$, $z^{\prime}(t)<0$, and (15) holds, there should exist a positive constant $a$ such that

$$
\lim _{t \rightarrow \infty} z(t)=a
$$

On the other hand, if $z^{(n-1)}(t)>0$ and $z^{(n)}(t) \leq 0$, there exists a constant $b \geq 0$ such that

$$
\lim _{t \rightarrow \infty} z^{(n-1)}(t)=b \geq 0 .
$$

Hence,

$$
\lim _{t \rightarrow \infty} z^{(i)}(t)=0
$$

for $i=1,2, \ldots, n-1$. Integrating (19) from $t$ to $\infty$ and using the fact that the limit

$$
\lim _{t \rightarrow \infty} r(t)\left(z^{(n-1)}(t)\right)^{\alpha} \geq 0
$$


is finite, we have

$$
\begin{gathered}
-r(t)\left(z^{(n-1)}(t)\right)^{\alpha}-\frac{p_{0}{ }^{\beta}}{\tau_{*}} r(\tau(t))\left(z^{(n-1)}(\tau(t))\right)^{\alpha} \\
+\int_{t}^{\infty} \mathrm{Q}(s) z^{\beta}(\sigma(s)) \mathrm{d} s \leq 0 .
\end{gathered}
$$

Consequently,

$$
\begin{aligned}
-R(t) & {\left[\left(z^{(n-1)}(t)\right)^{\alpha}+\left(\left(\frac{p_{0}^{\beta}}{\tau_{*}}\right)^{1 / \alpha}\right)^{\alpha}\left(z^{(n-1)}(\tau(t))\right)^{\alpha}\right] } \\
+ & \int_{t}^{\infty} \mathrm{Q}(s) z^{\beta}(\sigma(s)) \mathrm{d} s \leq 0 .
\end{aligned}
$$

Assume first that $\alpha \leq 1$. Using the result due to Baculíková [7, Lemma 2.2], we obtain

$$
\begin{gathered}
\left(z^{(n-1)}(t)\right)^{\alpha}+\left(\left(\frac{p_{0}^{\beta}}{\tau_{*}}\right)^{1 / \alpha}\right)^{\alpha}\left(z^{(n-1)}(\tau(t))\right)^{\alpha} \\
\leq 2^{1-\alpha}\left[z^{(n-1)}(t)+\left(\frac{p_{0}^{\beta}}{\tau_{*}}\right)^{1 / \alpha} z^{(n-1)}(\tau(t))\right]^{\alpha} .
\end{gathered}
$$

Substituting (92) into (91), we have

$$
\begin{gathered}
-2^{1-\alpha} R(t)\left[z^{(n-1)}(t)+\left(\frac{p_{0}^{\beta}}{\tau_{*}}\right)^{1 / \alpha} z^{(n-1)}(\tau(t))\right]^{\alpha} \\
+\int_{t}^{\infty} \mathrm{Q}(s) z^{\beta}(\sigma(s)) \mathrm{d} s \leq 0,
\end{gathered}
$$

which yields

$$
\begin{gathered}
-\left[z^{(n-1)}(t)+\left(\frac{p_{0}^{\beta}}{\tau_{*}}\right)^{1 / \alpha} z^{(n-1)}(\tau(t))\right]^{\alpha} \\
\leq-\frac{1}{2^{1-\alpha} R(t)} \int_{t}^{\infty} Q(s) z^{\beta}(\sigma(s)) \mathrm{d} s .
\end{gathered}
$$

Therefore,

$$
\begin{gathered}
-\left[z^{(n-1)}(t)+\left(\frac{p_{0}{ }^{\beta}}{\tau_{*}}\right)^{1 / \alpha} z^{(n-1)}(\tau(t))\right] \\
+\left[\frac{1}{2^{1-\alpha} R(t)} \int_{t}^{\infty} Q(s) z^{\beta}(\sigma(s)) \mathrm{d} s\right]^{1 / \alpha} \leq 0 .
\end{gathered}
$$

Integrate (95) $n-2$ times from $t$ to $\infty$ and then one more time from $t_{1}$ to $\infty$. Using (88) and changing the order of integration, we obtain

$$
\int_{t_{1}}^{\infty} \frac{\left(\xi-t_{1}\right)^{n-2}}{(n-2) !}\left[\frac{1}{2^{1-\alpha} R(\xi)} \int_{\xi}^{\infty} \mathrm{Q}(s) z^{\beta}(\sigma(s)) \mathrm{d} s\right]^{1 / \alpha} \mathrm{d} \xi<\infty .
$$

Inequality (96) yields

$$
\int_{t_{1}}^{\infty} \xi^{n-2}\left[\frac{1}{R(\xi)} \int_{\xi}^{\infty} Q(s) \mathrm{d} s\right]^{1 / \alpha} \mathrm{d} \xi<\infty,
$$

which contradicts (85).

For the case $\alpha>1$, one arrives at the contradiction with the assumptions of the theorem by using another auxiliary result obtained by Baculíková [7, Lemma 2.1]. Thus, we conclude that $z^{\prime}(t)>0$ eventually. The rest of the proof follows the same lines as in Theorem 3 and is omitted.

Combining the ideas exploited in the proofs of Theorems 4-6 and 15, one can derive the following results.

Theorem 16. Let $n \geq 3$ be odd, and let $0<\alpha=\beta \leq 1$. Assume that conditions $\left(H_{1}\right)$ and $\left(H_{2}\right)$ are satisfied, and there exist two functions $\eta_{1}, \eta_{2} \in C(\mathbb{\square}, \mathbb{R})$ satisfying (11). If (47), (48), and (85) hold, the conclusion of Theorem 3 remains intact.

Theorem 17. Let $n \geq 3$ be odd, and let $0<\beta \leq 1$. Assume that conditions $\left(H_{1}\right)$ and $\left(H_{2}\right)$ are satisfied, and there exist two numbers $\gamma, \lambda \in \mathfrak{R}$ as in Theorem 3 and two functions $\eta_{1}, \eta_{2} \in C(\mathbb{\square}, \mathbb{R})$ satisfying (51). If conditions (12), (13), and (85) are satisfied, the conclusion of Theorem 3 remains intact.

Theorem 18. Let $n \geq 3$ be odd, and let $0<\alpha=\beta \leq 1$. Assume that conditions $\left(H_{1}\right)$ and $\left(H_{2}\right)$ are satisfied, and there exist two functions $\eta_{1}, \eta_{2} \in C(\mathbb{\square}, \mathbb{R})$ satisfying (51). If conditions (59), (60), and (85) are satisfied, the conclusion of Theorem 3 remains intact.

\section{Examples and Discussion}

The following examples illustrate applications of some of theoretical results presented in the previous sections. In all the examples, $p_{0}$ is a constant such that $0 \leq p_{0}<\infty$.

Example 1. For $t \geq 1$, consider a fourth-order neutral differential equation

$$
\left(\mathrm{e}^{t}\left(x(t)+p_{0} x(t-2)\right)^{\prime \prime \prime}\right)^{\prime}+\frac{1}{16}\left(1+p_{0} \mathrm{e}\right) \mathrm{e}^{t-1 / 2} x(t-1)=0 .
$$

Let $\eta_{1}(t)=t-3$ and $\eta_{2}(t)=t+1$. An application of Theorem 6 yields that every solution $x(t)$ of (98) is either oscillatory or satisfies (14). As a matter of fact, $x(t)=\mathrm{e}^{-t / 2}$ is an exact solution to (98) satisfying (14).

Example 2. For $t \geq 1$, consider a fourth-order neutral differential equation

$$
\begin{gathered}
\left(\mathrm{e}^{t}\left(x(t)+p_{0} x(t+2 \pi)\right)^{\prime \prime \prime}\right)^{\prime}+2 \sqrt{10}\left(1+p_{0} \mathrm{e}^{2 \pi}\right) \\
\times \mathrm{e}^{t+\arcsin \sqrt{10} / 10} x\left(t-\arcsin \frac{\sqrt{10}}{10}\right)=0 .
\end{gathered}
$$

Let $\eta_{1}(t)=t-3$ and $\eta_{2}(t)=\eta_{3}(t)=t+3 \pi$. Using Theorem 8 , we deduce that (99) is oscillatory. It is not hard to verify that one oscillatory solution of this equation is $x(t)=\mathrm{e}^{t} \sin t$. 
Example 3. For $t \geq 1$, consider a third-order neutral differential equation

$$
\left(\mathrm{e}^{t}\left(x(t)+p_{0} x(t-2)\right)^{\prime \prime}\right)^{\prime}+\frac{9}{8}\left(1+p_{0} \mathrm{e}^{3}\right) \mathrm{e}^{t-3 / 2} x(t-1)=0 .
$$

Let $\eta(t)=t+1$. It follows from Theorem 14 that every solution $x(t)$ of (100) is either oscillatory or satisfies (14). In fact, one solution of this equation satisfying (14) is $x(t)=\mathrm{e}^{-t / 2}$.

Remark 4. In the case of (2), oscillation criteria established in this paper complement theorems reported by Zhang et al. [23, 25 ] because our criteria apply also in the case where $\sigma(t) \geq t$ and $\beta>\alpha$. On the other hand, our results for (1) supplement those reported by Baculíková and Džurina [10], Baculíková et al. [11], and Xing et al. [22] since our theorems can be applied if $\alpha \neq \beta$ and (3) holds.

Remark 5. By using inequality

$$
x_{1}^{\beta}+x_{2}^{\beta} \geq 2^{1-\beta}\left(x_{1}+x_{2}\right)^{\beta}
$$

which holds for any $\beta \geq 1$ and for all $x_{1}, x_{2} \in[0, \infty)$, results reported in this paper can be extended to (1) for all $\beta \in \Re$ which satisfy $\beta>1$. In this case, one has to replace $Q(t):=\min \{q(t), q(\tau(t))\}$ with a function $Q(t):=$ $2^{1-\beta} \min \{q(t), q(\tau(t))\}$ and proceed as above.

Remark 6. Our main assumptions on functional arguments do not specify whether $\tau(t)$ is a delayed or an advanced argument. Remarkably, $\sigma(t)$ can even switch its nature between an advanced and delayed argument. However, as in the paper by Baculíková and Džurina [10, condition $\left.\left(H_{3}\right)\right]$, such flexibility is achieved at the cost of requiring that the function $\tau$ is monotonic and satisfies $\tau \circ \sigma=\sigma \circ \tau$. The question regarding the analysis of the asymptotic behavior of solutions to (1) with other methods that do not require these assumptions remains open at the moment.

\section{Conflict of Interests}

The authors declare that they have no competing interests and no financial issues to disclose.

\section{Acknowledgment}

The authors thank anonymous referees for the careful reading of the paper and for pointing out several inaccuracies in the text.

\section{References}

[1] C. H. Ou and J. S. W. Wong, "Oscillation and non-oscillation theorems for superlinear Emden-Fowler equations of the fourth order," Annali di Matematica Pura ed Applicata. Series IV, vol. 183, no. 1, pp. 25-43, 2004.

[2] J. S. W. Wong, "On the generalized Emden-Fowler equation," SIAM Review, vol. 17, pp. 339-360, 1975.
[3] R. P. Agarwal, L. Berezansky, E. Braverman, and A. Domoshnitsky, Nonoscillation Theory of Functional Differential Equations with Applications, Springer, New York, NY, USA, 2012.

[4] R. P. Agarwal, M. Bohner, and W.-T. Li, Nonoscillation and Oscillation: Theory for Functional Differential Equations, vol. 267, Marcel Dekker, New York, NY, USA, 2004.

[5] R. P. Agarwal, S. R. Grace, and D. O'Regan, Oscillation Theory for Difference and Functional Differential Equations, Kluwer Academic, Dordrecht, The Netherlands, 2000.

[6] G. S. Ladde, V. Lakshmikantham, and B. G. Zhang, Oscillation Theory of Differential Equations with Deviating Arguments, vol. 110, Marcel Dekker, New York, NY, USA, 1987.

[7] B. Baculíková, "Properties of third-order nonlinear functional differential equations with mixed arguments," Abstract and Applied Analysis, vol. 2011, Article ID 857860, 15 pages, 2011.

[8] B. Baculíková and J. Džurina, "Oscillation theorems for second order neutral differential equations," Computers \& Mathematics with Applications, vol. 61, no. 1, pp. 94-99, 2011.

[9] B. Baculíková and J. Džurina, "Oscillation theorems for secondorder nonlinear neutral differential equations," Computers \& Mathematics with Applications, vol. 62, no. 12, pp. 4472-4478, 2011.

[10] B. Baculíková and J. Džurina, "Oscillation theorems for higher order neutral differential equations," Applied Mathematics and Computation, vol. 219, no. 8, pp. 3769-3778, 2012.

[11] B. Baculíková, J. Džurina, and T. Li, "Oscillation results for evenorder quasilinear neutral functional differential equations," Electronic Journal of Differential Equations, no. 143, pp. 1-9, 2011.

[12] J. Džurina and B. Baculíková, "Oscillation and asymptotic behavior of higher-order nonlinear differential equations," International Journal of Mathematics and Mathematical Sciences, vol. 2012, Article ID 951898, 9 pages, 2012.

[13] M. Hasanbulli and Yu. V. Rogovchenko, "Asymptotic behavior of nonoscillatory solutions to $n$-th order nonlinear neutral differential equations," Nonlinear Analysis: Theory, Methods \& Applications, vol. 69, no. 4, pp. 1208-1218, 2008.

[14] M. Hasanbulli and Yu. V. Rogovchenko, "Oscillation criteria for second order nonlinear neutral differential equations," Applied Mathematics and Computation, vol. 215, no. 12, pp. 4392-4399, 2010.

[15] Y. Kitamura and T. Kusano, "Oscillation of first-order nonlinear differential equations with deviating arguments," Proceedings of the American Mathematical Society, vol. 78, no. 1, pp. 64-68, 1980.

[16] T. Li, R. P. Agarwal, and M. Bohner, "Some oscillation results for second-order neutral differential equations," The Journal of the Indian Mathematical Society, vol. 79, no. 1-4, pp. 97-106, 2012.

[17] T. Li, Z. Han, P. Zhao, and S. Sun, "Oscillation of even-order neutral delay differential equations," Advances in Difference Equations, vol. 2010, Article ID 184180, 2010.

[18] T. Li, Yu. V. Rogovchenko, and C. Zhang, "Oscillation of secondorder neutral differential equations," Funkcialaj Ekvacioj, vol. 56, no. 1, pp. 111-120, 2013.

[19] T. Li and E. Thandapani, "Oscillation of solutions to odd-order nonlinear neutral functional differential equations," Electronic Journal of Differential Equations, no. 23, pp. 1-12, 2011.

[20] Ch. G. Philos, "A new criterion for the oscillatory and asymptotic behavior of delay differential equations," Bulletin de l'Académie Polonaise des Sciences, vol. 39, pp. 61-64, 1981. 
[21] Ch. G. Philos, "On the existence of nonoscillatory solutions tending to zero at $\infty$ for differential equations with positive delays," Archiv der Mathematik, vol. 36, no. 2, pp. 168-178, 1981.

[22] G. Xing, T. Li, and C. Zhang, "Oscillation of higher-order quasilinear neutral differential equations," Advances in Difference Equations, vol. 2011, article 45, p. 10, 2011.

[23] C. Zhang, R. P. Agarwal, M. Bohner, and T. Li, "New results for oscillatory behavior of even-order half-linear delay differential equations," Applied Mathematics Letters, vol. 26, no. 2, pp. 179183, 2013.

[24] C. Zhang, T. Li, R. P. Agarwal, and M. Bohner, "Oscillation results for fourth-order nonlinear dynamic equations," Applied Mathematics Letters, vol. 25, no. 12, pp. 2058-2065, 2012.

[25] C. Zhang, T. Li, B. Sun, and E. Thandapani, "On the oscillation of higher-order half-linear delay differential equations," Applied Mathematics Letters, vol. 24, no. 9, pp. 1618-1621, 2011. 


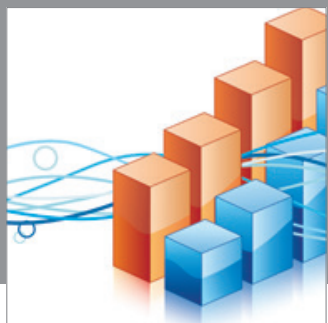

Advances in

Operations Research

mansans

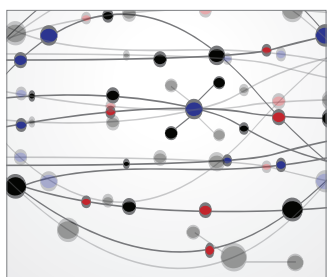

The Scientific World Journal
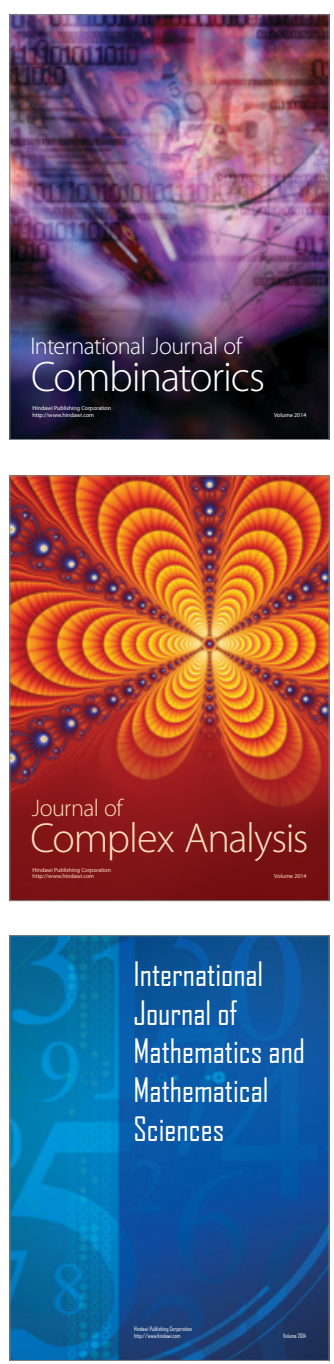
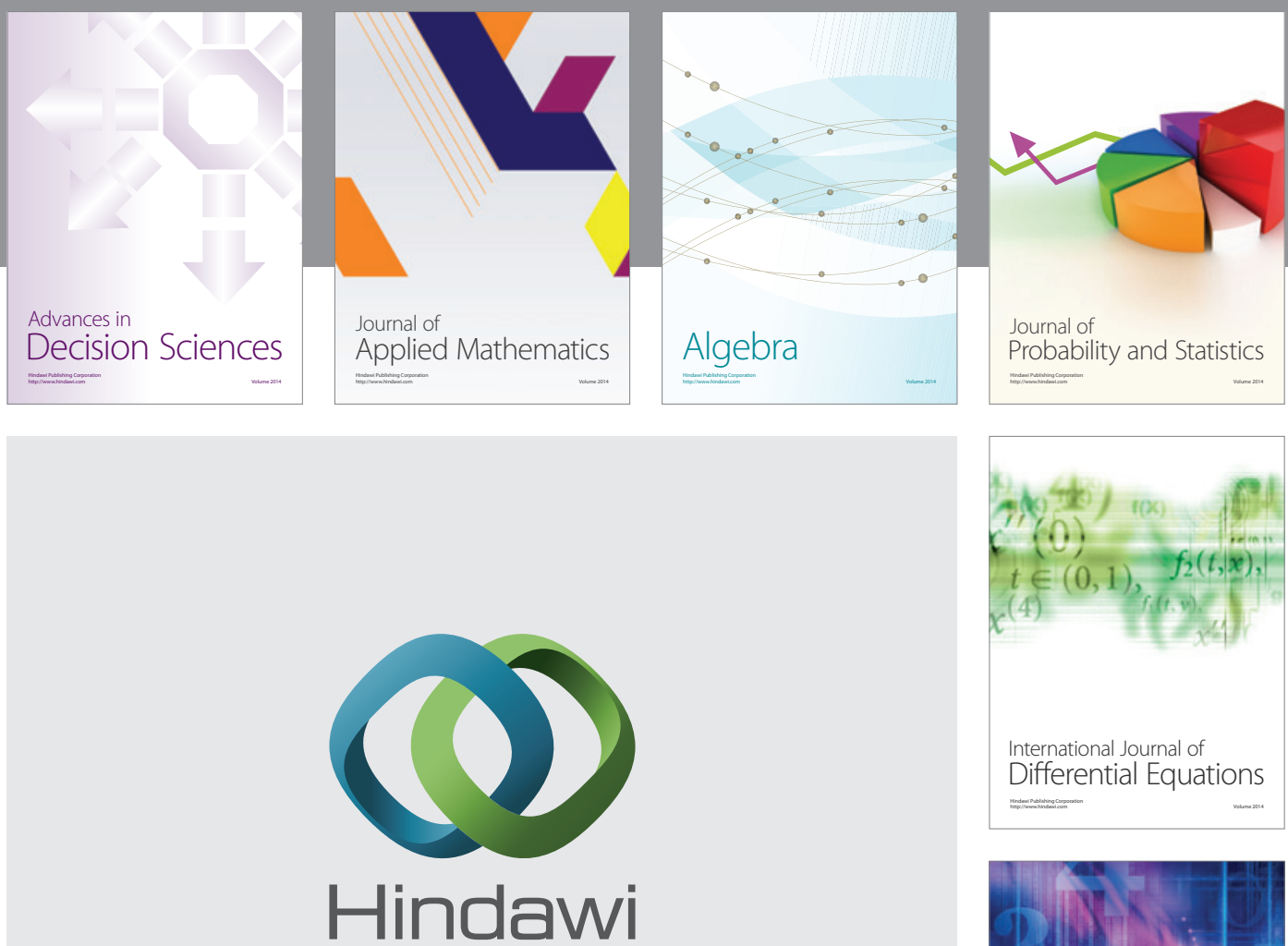

Submit your manuscripts at http://www.hindawi.com
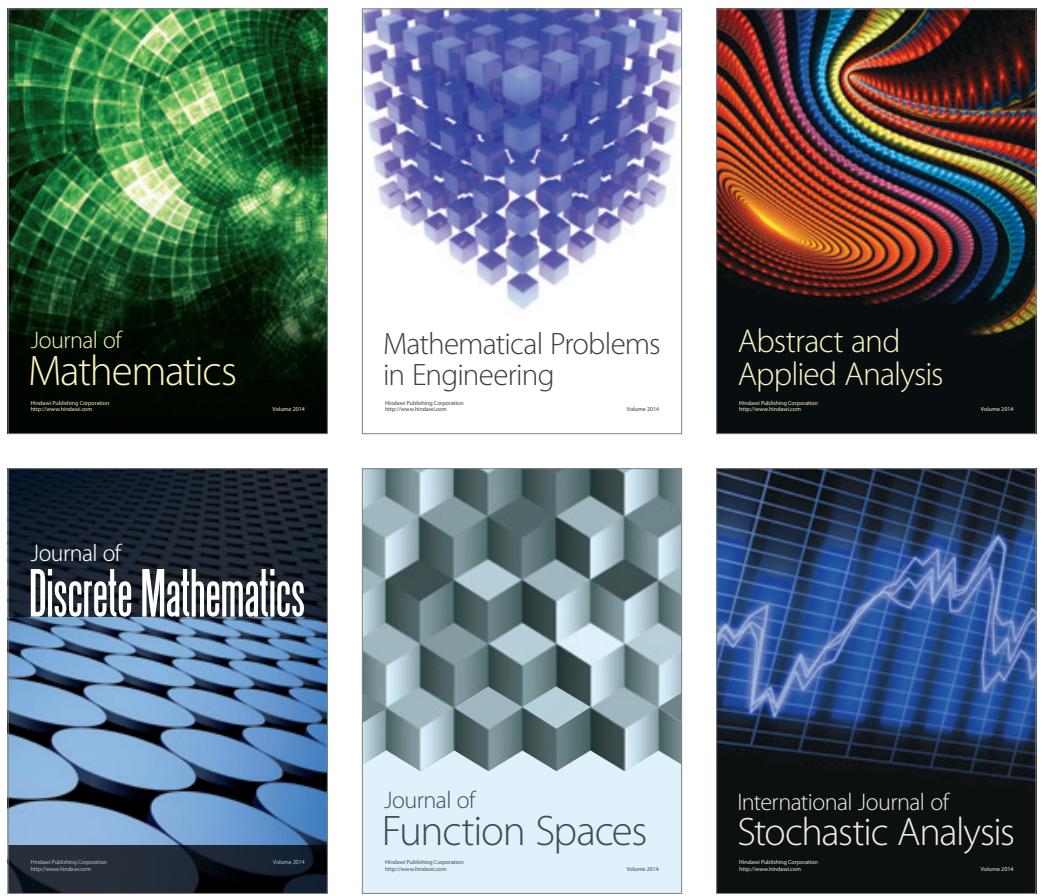

Journal of

Function Spaces

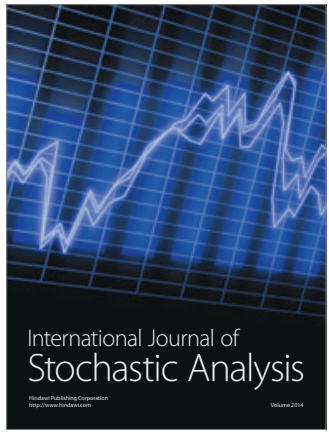

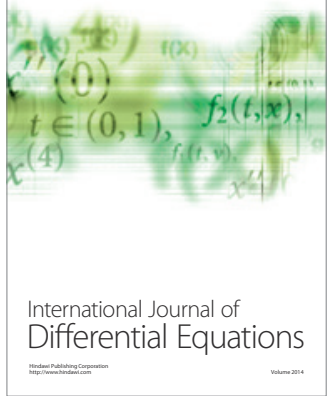
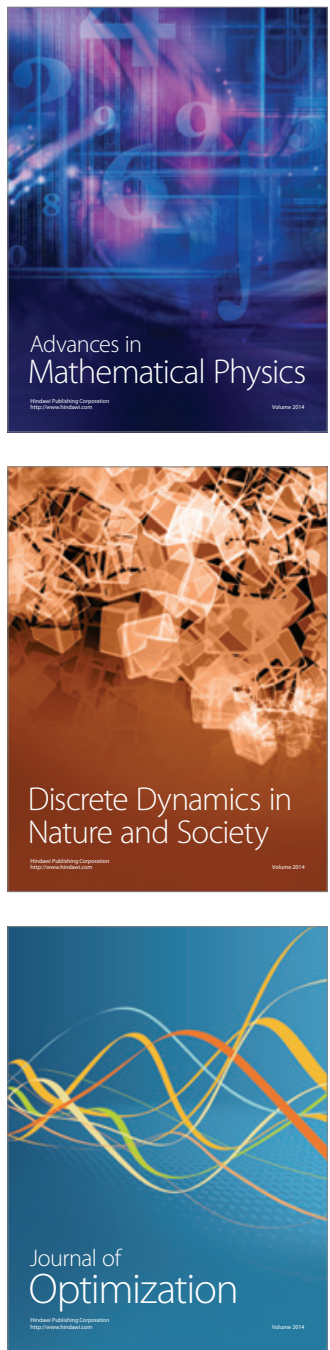